\title{
Improving Optimal Terminal Value Replicating Portfolios
}

\author{
Jan Natolski and Ralf Werner
}

\begin{abstract}
Currently, several large life insurance companies apply the replicating portfolio technique for valuation and risk management of their liabilities. In [7], the two most common approaches, cash-flow matching and terminal value matching, have been investigated from a theoretical perspective and it has been shown that optimal terminal value replicating portfolios are not suitable to replicate liability cash-flows by construction. Thus, their usage for asset liability management is rather restricted, especially for out-of-sample cash profiles of liabilities. In this paper, we therefore enhance the terminal value approach by an additional linear regression of the corresponding optimal dynamic numéraire strategy to overcome this drawback. We show that terminal value matching together with an approximated dynamic strategy has in-sample and out-of-sample performance very close to the optimal cashflow matching portfolio and, due to computational advantages, can thus be used as an alternative for cash-flow matching, especially in risk and asset liability management.
\end{abstract}

\section{Introduction}

In the last years, market consistent valuation has become the standard approach toward risk management of life insurance policies, see for example [3]. Due to the complexity of life insurance contracts, most academics and practitioners resort to Monte Carlo methods for valuation purposes. However, the difficulty is to find a computationally efficient yet sufficiently accurate algorithm. For instance, contracts may include surrender options, which allow the policy holder every year to cancel the contract and withdraw the value of her account. In this context, $[1,2]$ and several

\author{
J. Natolski $\cdot$ R. Werner $(\bowtie)$ \\ University of Augsburg, Universitätsstraße 14, 86159 Augsburg, Germany \\ e-mail: ralf.werner@math.uni-augsburg.de \\ J. Natolski \\ e-mail: jan.natolski@math.uni-augsburg.de \\ (C) The Author(s) 2015 \\ $\mathrm{K}$. Glau et al. (eds.), Innovations in Quantitative Risk Management, \\ Springer Proceedings in Mathematics \& Statistics 99, \\ DOI 10.1007/978-3-319-09114-3_16
}


other authors therefore resort to the well-known least squares Monte Carlo approach, which was originally introduced by [6] to price American options. In contrast, [9] first suggested valuation of with-profits guaranteed annuity options, which are typical life insurance products, via static replicating portfolios. To hedge against interest rate risk, a portfolio is built of vanilla swaptions and a remarkably good fit of the market value of annuity options is obtained. The purpose of constructing a replicating portfolio is to approximate the liability cash-flows of an insurance company by a portfolio formed by a finite number of selected financial instruments. If the approximation is accurate, one obtains a good estimate of the market value of liabilities from the fair value of the replicating portfolio. In current literature, two portfolio construction approaches stand out. The first one aims to match liability cash-flows and cash-flows of the replicating portfolio at each time point. The second one is less restrictive as it only demands that accrued terminal values of the cash-flows match well at some final time horizon $T$.

For risk purposes, insurance companies want to compute the fair value of their assets and liabilities, i.e., the market consistent embedded value (MCEV) under shifted market conditions now or one year in the future. More precisely, having found a replicating portfolio which matches the fair value of liabilities under current market conditions, one performs instantaneous shocks on known parameters (such as volatility, forward rate curve, etc.) and checks if fair values are still matched. This is commonly referred to as a comparison of sensitivities between the fair value of the replicating portfolio and the fair value of liabilities. If sensitivities are similar, it is usually assumed that fair values will be roughly matched one year in the future even if rare events in the $99.5 \%$ quantile take place. For instance, this is the motivation for [4] to put additional constraints in the optimization problem to guarantee that fair values are close to one another under various stress scenarios. Figure 1 illustrates the dependence between initial asset prices and the fair value of liabilities and a replicating portfolio. It can be observed that fair values are close to each other and behave quite similar, but not fully identical.

For the purpose of improving terminal value matching, we start with the setup as given in [7], that is, we consider the cash-flow matching problem and the terminal value matching problem as proposed in [9] and [8], respectively, and relax the requirement of static replication by allowing for dynamic investment strategies in the numéraire asset. We briefly review the theoretical results derived therein, before we investigate in more detail the benefit of our approach based on market scenarios generated by an insurance company: First, we compare the in-sample and out-ofsample performance of the two replicating portfolios. Then, in the main contribution of this article, we take a closer look at the optimal dynamic investment strategy and approximate it by a time-dependent linear combination of the replicating assets. In our particular example, the approximation turns out to be remarkably accurate as in-sample and out-of sample tests will show. 


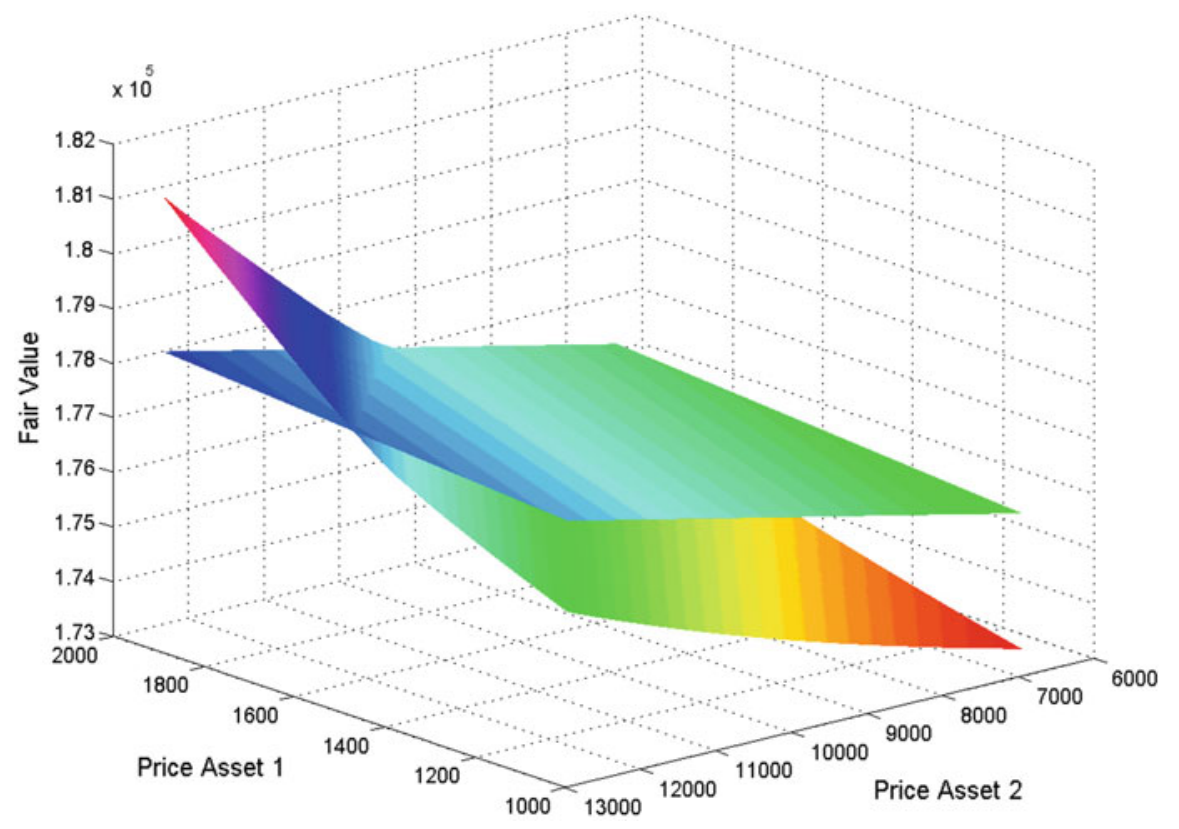

Fig. 1 Fair value of liabilities and of the replicating portfolio depending on initial asset prices

\section{The Mathematical Setup}

This setup roughly follows that of [3]: Let $\left(\Omega, \mathscr{F},\left(\mathscr{F}_{t}\right)_{t \in \mathscr{T}}, \mathbb{Q}\right)$ be a filtered probability space ${ }^{1}$ in discrete time $\mathscr{T}:=\{t=0,1 \ldots, T\}$ with risk-neutral measure $\mathbb{Q}$. On this probability space, we introduce a frictionless, arbitrage-free financial market as follows.

- Let $\left(R_{t}^{F}\right)_{t} \in \mathbb{R}^{n}, t \in \mathscr{T}$ be Markovian financial risk factors (e.g., interest rates).

- Let $\left(R_{t}^{L}\right)_{t} \in \mathbb{R}^{l}, t \in \mathscr{T}$ be Markovian risk factors, independent of $\left(R_{t}^{F}\right)_{t \in \mathscr{T}}$, affecting the liabilities of an insurance company (e.g., mortality rates).

- $\left(R_{t}^{F}\right)_{t \in \mathscr{T}}$ and $\left(R_{t}^{L}\right)_{t \in \mathscr{T}}$ generate filtrations $\left(\mathscr{F}_{t}^{F}\right)_{t \in \mathscr{T}}$ and $\left(\mathscr{F}_{t}^{L}\right)_{t \in \mathscr{T}}$ respectively. We assume $\mathscr{F}_{t}=\mathscr{F}_{t}^{F} \vee \mathscr{F}_{t}^{L}, \forall t \in \mathscr{T}$.

- There are $m$ financial assets, an $\mathbb{R}^{d_{1}}$-valued $\left(\mathscr{F}_{t}^{F}\right)$-adapted process $\left(D_{t}^{F}\right)_{t \in \mathscr{T}}$ (e.g., risk factors, book values, moving averages, etc.) and a function $C_{i}^{F}:\{1, \ldots, T\} \times$ $\mathbb{R}^{d_{1}} \mapsto \mathbb{R}$ for each asset $i$ such that $C_{i}^{F}\left(t, D_{t}^{F}\right)$ is the cash payment of asset $i$ at time $t$. At $T$, this cash payment represents the remaining value of the asset. $D^{L}$ and $C^{L}$ are defined analogously; however, liabilities may also depend on the financial risk factors $D^{F}$, i.e., $C^{L}=C^{L}\left(t, D_{t}^{F}, D_{t}^{L}\right)$.

\footnotetext{
${ }^{1}$ Similarly to [7] we assume that all technical requirements are fulfilled (square integrability, completeness of filtration, ...).
} 
- $\left(N_{t}\right)_{t \in \mathscr{T}}$ denotes the numéraire (with initial value $N_{0}=1$, paying no intermediate cash-flows) which is used in the dynamic investment strategy. We assume that $N_{T}$ is paid as a cash-flow at the final time horizon. For convenience, let us write $C_{0}^{F}\left(t, D_{t}^{F}\right)$ for the cash payment of the numéraire at time $t$, that is

$$
C_{0}^{F}\left(t, D_{t}^{F}\right)=\left\{\begin{array}{ll}
0, & t=1, \ldots, T-1 \\
N_{T}, & t=T
\end{array} .\right.
$$

Next, we review the two most commonly used approaches for the construction of a replicating portfolio.

\section{The Theory of Replicating Portfolios}

\subsection{Cash-Flow Matching}

One possibility proposed by [9] is to look for a portfolio $\left(\alpha_{\mathrm{opt}}^{0}, \ldots, \alpha_{\mathrm{opt}}^{m}\right) \in \mathbb{R}^{m+1}$, which solves the optimization problem ${ }^{2}$

$$
\min _{\alpha \in \mathbb{R}^{m+1}} \sum_{t=1}^{T}\left[\mathbb{E}^{\mathbb{Q}}\left(\left[\frac{C^{L}\left(t, D^{F}{ }_{t}, D^{L} t\right)}{N_{t}}-\sum_{i=0}^{m} \alpha^{i} \frac{C^{F}{ }_{i}\left(t, D^{F}{ }_{t}\right)}{N_{t}}\right]^{2}\right)\right]^{\frac{1}{2}} \cdot \quad\left(\mathrm{RP}_{\mathrm{CF}}\right)
$$

The objective function penalizes the difference between two cash payments at each time $t$. The role of the discounting factor $1 / N_{t}$ is to assign equal weight to mismatches of equal size in terms of their discounted value. An alternative approach is discounted terminal value matching.

\subsection{Discounted Terminal Value Matching}

The terminal value of a cash-flow is obtained by summing all cash payments accrued to the terminal time $T$ with the risk-free interest rate. By discounted terminal value, we mean the accrued terminal value discounted to the present. In mathematical notation, the discounted accrued liability cash-flow and the discounted accrued cash-flow of a replicating portfolio $\alpha=\left(\alpha^{0}, \ldots, \alpha^{m}\right) \in \mathbb{R}^{m+1}$ are given by

\footnotetext{
2 The existence of a minimum has been shown in [7].
} 


$$
\begin{aligned}
\tilde{A}^{L} & :=\sum_{t=1}^{T} \frac{C^{L}\left(t, D_{t}^{F}, D_{t}^{L}\right)}{N_{t}}, \\
\tilde{A}^{F}(\alpha) & :=\sum_{t=1}^{T}\left[\sum_{i=0}^{m} \alpha^{i} \frac{C_{i}^{F}\left(t, D_{t}^{F}\right)}{N_{t}}\right] .
\end{aligned}
$$

The observation that although two cash-flows may have entirely different cash payment profiles, they still have the same fair value, leads to the alternative optimization proble $^{3}$

$$
\min _{\alpha \in \mathbb{R}^{m+1}}\left[\mathbb{E}^{\mathbb{Q}}\left(\left[\tilde{A}^{L}-\tilde{A}^{F}(\alpha)\right]^{2}\right)\right]^{\frac{1}{2}}
$$

Originally, this problem was introduced by [8] with the difference that they considered non-discounted terminal values.

\section{Equivalence of Cash-Flow Matching and Discounted Terminal Value Matching}

Next, we recall the connection between $\left(\mathrm{RP}_{\mathrm{CF}}\right)$ and $\left(\mathrm{RP}_{\mathrm{TV}}\right)$ as established in [7]. If the numéraire asset can be bought or sold at any time, problems $\left(\mathrm{RP}_{\mathrm{CF}}\right)$ and $\left(\mathrm{RP}_{\mathrm{TV}}\right)$ are practically the same. The brief explanation is that cash-flow mismatches can be laid off by an appropriate strategy in this asset. These mismatches then sum up to the discounted terminal value mismatch and thus problems $\left(\mathrm{RP}_{\mathrm{CF}}\right)$ and $\left(\mathrm{RP}_{\mathrm{TV}}\right)$ are intimately linked.

In more detail, suppose that the insurance company is allowed to invest and finance cash-flows from trading the numéraire asset at all times $t=1, \ldots, T$. Define the following linear space of processes

$$
\mathscr{A}=\left\{\left(\delta_{t}\right)_{t=1, \ldots, T}: \forall t=1, \ldots, T-1, \delta_{t} \in \mathscr{L}^{2}\left(\mathscr{F}_{t}\right), \quad \sum_{t=1}^{T} \delta_{t}=0\right\}
$$

Any process from this space represents an adapted strategy of investments in or borrowing from the numéraire asset, so $\delta_{t}$ is the number of assets bought or sold short at time $t$. Here, $\delta_{t}>0$ is interpreted as a purchase, which corresponds to a negative cash-flow for the insurer and $\delta_{t}<0$ as a sale, which corresponds to a positive cash-flow. The condition $\sum_{t=1}^{T} \delta_{t}=0$ ensures that strategies have zero discounted terminal value. Note that strategies from $\mathscr{A}$ are not necessarily predictable. At each time point, the insurer can incorporate all information available at that time to make a decision on the trade $\delta_{t}$. Only at $T$ is the insurer bound to clear the balance thus making $\delta_{T}$ predictable.

\footnotetext{
${ }^{3}$ For the existence of a minimum, see [7].
} 
The introduction of such strategies turns out to be the key link between problems $\left(\mathrm{RP}_{\mathrm{CF}}\right)$ and $\left(\mathrm{RP}_{\mathrm{TV}}\right)$ : The discounted terminal value $\tilde{A}^{F}(\alpha, \delta)$ corresponding to an investment strategy $(\alpha, \delta)$ with $\alpha \in \mathbb{R}^{m+1}, \delta \in \mathscr{A}$ is given by

$$
\tilde{A}^{F}(\alpha, \delta)=\tilde{A}^{F}(\alpha)
$$

where

$$
\tilde{A}^{F}(\alpha, \delta):=\sum_{t=1}^{T}\left[\sum_{i=0}^{m} \alpha^{i} \frac{C_{i}^{F}\left(t, D_{t}^{F}\right)}{N_{t}}\right]-\sum_{t=1}^{T} \delta_{t} .
$$

In other words, the discounted terminal value only depends on the initial portfolio $\alpha^{0}, \alpha^{1}, \ldots, \alpha^{m}$ in the assets. Thus, we write $\tilde{A}^{F}(\alpha)$ instead of $\tilde{A}^{F}(\alpha, \delta)$. We say that two investment strategies $(\alpha, \delta)$ and $(\beta, \hat{\delta})$ with $\alpha, \beta \in \mathbb{R}^{m+1}, \delta, \hat{\delta} \in \mathscr{A}$ are FV-equivalent iff

$$
\alpha=\beta \text {. }
$$

Note that due to the above, initial portfolios of two FV-equivalent investment strategies have equal fair value, as they produce identical discounted terminal values.

Based on the extension from static portfolios to partially dynamic strategies, we define corresponding optimization problems,

$$
\inf _{\alpha \in \mathbb{R}^{m+1}, \delta \in \mathscr{A}} \sum_{t=1}^{T}\left[\mathbb{E}^{\mathbb{Q}}\left(\left[\frac{C^{L}\left(t, D^{F}{ }_{t}, D^{L}{ }_{t}\right)}{N_{t}}-\left(\sum_{i=0}^{m} \alpha^{i} \frac{C^{F}{ }_{i}\left(t, D^{F}{ }_{t}\right)}{N_{t}}-\delta_{t}\right)\right]^{2}\right)\right]^{\frac{1}{2}}, \quad\left(\mathrm{GRP}_{\mathrm{CF}}\right)
$$

the generalized cash-flow matching problem and

$$
\inf _{\alpha \in \mathbb{R}^{m+1}, \delta \in \mathscr{A}}\left[\mathbb{E}^{\mathbb{Q}}\left(\left[\tilde{A}^{L}-\tilde{A}^{F}(\alpha)\right]^{2}\right)\right]^{\frac{1}{2}}
$$

the generalized discounted terminal value matching problem. Based on the following two additional weak assumptions, the main results follow.

Assumption 1 The matrix $\mathbb{E}^{\mathbb{Q}}\left(Q^{F}\right)$ is positive definite, where

$$
Q^{F}:=\left(\tilde{A}_{i}^{F} \tilde{A}_{j}^{F}\right)_{i, j=0, \ldots, m}
$$

with discounted terminal value $\tilde{A}_{i}^{F}$ of the cash-flow generated by asset $i$ given as

$$
\tilde{A}_{i}^{F}:=\sum_{t=1}^{T} \frac{C_{i}^{F}\left(t, D_{t}^{F}\right)}{N_{t}}
$$


Assumption 2 Let $\alpha_{\mathrm{opt}}=\left(\alpha_{\mathrm{opt}}^{0}, \alpha_{\mathrm{opt}}^{1}, \ldots, \alpha_{\mathrm{opt}}^{m}\right)$ be the solution to $\left(\mathrm{RP}_{\tilde{\mathrm{TV}}}\right)$. The cash-flow mismatch $C^{L}\left(T, D_{T}^{F}, D_{T}^{L}\right)-\sum_{i=0}^{m} \alpha_{\mathrm{opt}}^{i} C_{i}^{F}\left(T, D_{T}^{F}\right)$ is not $\mathscr{F}_{T-1}$ measurable.

The following properties of the two optimization problems and their connections were derived in [7].

\section{Properties of $\left(\mathrm{GRP}_{\mathrm{TV}}\right)$ and the relationship to $\left(\mathrm{RP}_{\mathrm{TV}}\right)$ :}

a. Under Assumption 1, the solution to $\left(\mathrm{RP}_{\tilde{\mathrm{TV}}}\right)$ exists, is unique and given by $\alpha_{\text {opt }}=\mathbb{E}^{\mathbb{Q}}\left(Q^{F}\right)^{-1} \mathbb{E}^{\mathbb{Q}}\left(\tilde{A}^{F} \tilde{A}^{L}\right)$. The set of solutions to $\left(\mathrm{GRP}_{\mathrm{TV}}\right)$ is the FV-equivalence class of the solution to $\left(\mathrm{RP}_{\mathrm{TV}}\right)$.

b. The optimal value of $\left(\mathrm{GRP}_{\tilde{\mathrm{TV}}}\right)$ is equal to the optimal value of $\left(\mathrm{RP}_{\mathrm{TV}}\right)$.

2. Properties of $\left(\mathrm{GRP}_{\mathrm{CF}}\right)$ and the relationship to $\left(\mathrm{RP}_{\mathrm{TV}}\right),\left(\mathrm{GRP}_{\mathrm{TV}}\right)$ and $\left(\mathrm{RP}_{\mathrm{CF}}\right)$ :

a. Under Assumptions 1 and 2, the solution to $\left(\mathrm{GRP}_{\mathrm{CF}}\right)$ exists and is unique with initial portfolio given by the solution to $\left(\mathrm{RP}_{\mathrm{TV}}\right)$ and strategy $\delta \in \mathscr{A}$ such that cash-flows are perfectly matched at times $t=1, \ldots, T-1$.

b. Under Assumption 1, the set of solutions to $\left(\mathrm{GRP}_{\mathrm{TV}}\right)$ is the equivalence class of the solution to $\left(\mathrm{GRP}_{\mathrm{CF}}\right)$.

c. The optimal value of $\left(\mathrm{GRP}_{\mathrm{CF}}\right)$ is smaller than or equal to the optimal value of $\left(\mathrm{RP}_{\mathrm{CF}}\right)$. Under Assumptions 1 and 2, equality is achieved iff for times $t=1, \ldots, T-1$ the liability cash-flow is perfectly replicated by the cashflow of the portfolio solving $\left(\mathrm{RP}_{\mathrm{TV}}\right)$.

\section{Fair values of $\left(\mathrm{GRP}_{\tilde{\mathrm{TV}}}\right)$ and $\left(\mathrm{GRP}_{\mathrm{CF}}\right)$ :}

a. The fair value of the solutions to $\left(\mathrm{GRP}_{\mathrm{TV}}\right)$ and the fair value of the solution to $\left(\mathrm{GRP}_{\mathrm{CF}}\right)$ are equal to the fair value of the liability cash-flow.

The main drawback of the generalized terminal value approach lies in the introduction of the dynamic strategy in the numéraire asset: as the optimal $\delta_{t}$ depend on the liability cash-flow (see Property 2.c above), this strategy is not available outof-sample to reproduce (unknown!) liability cash-flows. Although the main purpose of replicating portfolios in risk management is fair value replication, asset liability management usually requires cash-flow replication as well.

Therefore, the optimal numéraire strategy has to be estimated based on available information up to time $t$, which then in turn allows a reproduction of liability cashflows, even in a terminal value approach. The most simple approach toward this end is a standard linear regression of the optimal $\delta_{t}$ against the information available in time $t$. Besides the obvious usage of prices of financial instruments as explaining variables, any further available information (e.g., non-traded risk factors like interest rate, etc.) could in theory be used for this purpose.

Starting with the portfolio solving $\left(\mathrm{RP}_{\mathrm{TV}}\right)$, we compute $\left(\delta_{t}\right)_{t=1, \ldots, T}$ such that cash-flows are perfectly matched in-sample except for $T$. The idea is to approximate $\delta_{t}, t=1, \ldots T-1$ by an ordinary linear regression, that is 


$$
\begin{aligned}
& \hat{\delta}_{t}(a):=a_{t}^{1} \frac{C_{\mathrm{CA}}^{F}\left(t, D_{t}^{F}\right)}{N_{t}}+a_{t}^{2} \frac{C_{\mathrm{SP}}^{F}\left(t, D_{t}^{F}\right)}{N_{t}}+a_{t}^{3} \frac{C_{\mathrm{NK}}^{F}\left(t, D_{t}^{F}\right)}{N_{t}}, t=1, \ldots, T-1, \\
& \hat{\delta}_{T}(a):=-\sum_{t=1}^{T-1} \hat{\delta}_{t}
\end{aligned}
$$

where $a \in \mathbb{R}^{T-1 \times 3}$ solves the problem

$$
\min _{a \in \mathbb{R}^{T-1 \times 3}} \sum_{t=1}^{T}\left[\mathbb{E}^{\mathbb{Q}}\left(\left[\frac{C^{L}\left(t, D^{F} t, D^{L} t\right)}{N_{t}}-\left(\sum_{i=0}^{m} \alpha_{\mathrm{opt}}^{i} \frac{C^{F}{ }_{i}\left(t, D^{F} t\right)}{N_{t}}-\hat{\delta}_{t}(a)\right)\right]^{2}\right)\right]^{\frac{1}{2}} .
$$

In other words, we solve $\left(\mathrm{GRP}_{\mathrm{CF}}\right)$ with $\alpha_{\mathrm{opt}}^{0}, \ldots, \alpha_{\mathrm{opt}}^{m}$ fixed and optimal for $\left(\mathrm{RP}_{\mathrm{TV}}\right)$ and $\delta_{t}$ restricted to have the form above. Note that the parameters $\left(a_{t}^{1}, a_{t}^{2}, a_{t}^{3}\right)_{t=1, \ldots T-1}$ are known to the insurer at present. The hope is that the portfolio obtained from matching discounted terminal values together with dynamic investment strategy $\left(\hat{\delta}_{t}\right)_{t=1, \ldots, T}$ will produce at least a similar out-of-sample objective value as the static portfolio obtained from solving $\left(\mathrm{RP}_{\mathrm{CF}}\right)$.

\section{Example}

Based on financial market scenarios provided by a life insurer, we carry out some numerical analysis to compare the performance of the portfolios solving $\left(\mathrm{RP}_{\mathrm{CF}}\right)$ and $\left(\mathrm{RP}_{\mathrm{TV}}\right)$. The results above imply that in an in-sample test the terminal value technique will outperform the cash-flow matching technique. On the other hand, it is not clear what happens in an out-of-sample test. This will also depend on the robustness of both methods.

Since scenarios for liability cash-flows were unavailable, we implemented the model proposed by [5]. A policy holder pays an initial premium $P_{0}$, which is invested by the insurer in a corresponding portfolio of assets with value process $\left(A_{t}\right)_{t \in \mathbb{N}}$. The value of the contract $\left(L_{t}\right)_{t \in \mathbb{N}}$ now evolves according to the following recursive formula.

$$
L_{t+1}=L_{t}\left(1+\max \left(r_{G}, \rho\left(\frac{A_{t}-L_{t}}{L_{t}}-\gamma\right)\right)\right), \quad t=0,1, \ldots,
$$

where $r_{G}$ is the interest guaranteed to the policy holder, $\rho$ is the level of participation in market value earnings and $\gamma$ is a target buffer ratio.

To generate liability cash-flows, we assumed that starting in January 1998 the insurance company receives one client every year up to 2012. Each client pays an initial nominal premium of 10.000 Euros. All contracts run 15 years. At maturity, the value of the contract is paid to the policy holder generating a liability cash-flow. 
The portfolio in which the premia are invested consists of the Standard and Poors 500 index, the Nikkei 225 index and the cash account. We normed the values of the cash account, the S and P 500 and the Nikkei 225 so that all three have value 1 Euro in year 2012. Every year the portfolio is adjusted such that $80 \%$ of the value are invested in the cash account and $10 \%$ are invested in each index.

For the construction of the replicating portfolio, we chose the same three assets. Cash-flows are generated by selling or buying assets every year. Since we are constructing a static portfolio, the decision how many assets will be bought or sold each year in the future has to be made in the present. Hence, one may regard the replicating assets as $3 \times 15$ call options with strike 0 , one option for each index/cash account and each year. We chose the cash account as the numéraire asset in the market.

In order to make the evolution of contract values more sensitive to changes of financial asset prices, we assumed a low guaranteed interest rate of $r_{G}=2.0 \%$, a high participation ratio $\rho=0.75$ and a low target buffer ratio $\gamma=0.05$. From 1,000 scenarios, ${ }^{4}$ we chose to use 500 for the construction of the replicating portfolios and the remaining 500 for an out-of-sample performance test. The portfolio is constructed

Table 1 Optimal replicating portfolios (in thousand Euros) for problems $\left(\mathrm{RP}_{\mathrm{CF}}\right)$ and $\left(\mathrm{RP}_{\mathrm{TV}}\right)$ and their fair value

\begin{tabular}{|c|c|c|c|c|c|c|}
\hline \multirow[b]{2}{*}{ Year } & \multicolumn{3}{|c|}{ Cash-flow match } & \multicolumn{3}{|c|}{ Terminal value match } \\
\hline & Cash account & S\&P & Nikkei & Cash account & S\&P & Nikkei \\
\hline Total initial position & 173.010 & 2.688 & 0.886 & 178.091 & 11.174 & -12.047 \\
\hline Fair value & \multicolumn{3}{|l|}{176.6} & \multicolumn{3}{|l|}{177.2} \\
\hline 2012 & 14.089 & 0 & 0 & 0 & 3.062 & -5.530 \\
\hline 2013 & 13.518 & 0.042 & -0.005 & 0 & 2.739 & -2.969 \\
\hline 2014 & 13.141 & 0.052 & -0.125 & 0 & 4.511 & -8.693 \\
\hline 2015 & 12.757 & 0.171 & -0.091 & 0 & -1.790 & 3.402 \\
\hline 2016 & 12.719 & 0.237 & -0.137 & 0 & -0.745 & -3.480 \\
\hline 2017 & 12.728 & 0.261 & -0.038 & 0 & -0.093 & -0.459 \\
\hline 2018 & 11.685 & 0.250 & 0.046 & 0 & -1.600 & 4.009 \\
\hline 2019 & 11.300 & 0.304 & 0.081 & 0 & 1.269 & -8.352 \\
\hline 2020 & 10.964 & 0.212 & 0.065 & 0 & 0.123 & 7.723 \\
\hline 2021 & 10.606 & 0.196 & 0.131 & 0 & 1.013 & 2.730 \\
\hline 2022 & 10.308 & 0.208 & 0.169 & 0 & 2.139 & -1.091 \\
\hline 2023 & 10.155 & 0.269 & 0.287 & 0 & 2.179 & -1.842 \\
\hline 2024 & 9.847 & 0.184 & 0.191 & 0 & -1.522 & 4.333 \\
\hline 2025 & 9.645 & 0.152 & 0.143 & 0 & 0.828 & -0.190 \\
\hline 2026 & 9.549 & 0.150 & 0.168 & 178.091 & -0.939 & -1.637 \\
\hline
\end{tabular}

The sample fair value of liabilities for the first 500 scenarios is $1.76 \times 10^{5}$ Euros

\footnotetext{
${ }^{4}$ As scenarios were provided by a life insurance company, only this restricted number of scenarios was available. Scenario paths for the Nikkei and the S\&P indices as well as the cash account
} 
Table 2 Values of the objective function in $\left(\mathrm{RP}_{\mathrm{CF}}\right)$ for optimal portfolios to $\left(\mathrm{RP}_{\mathrm{CF}}\right)$ and $\left(\mathrm{RP}_{\mathrm{TV}}\right)$ relative to the fair value of liabilities

\begin{tabular}{l|l|l}
\hline & In-sample (\%) & Out-of-sample (\%) \\
\hline Cash-flow & 8.72 & 9.23 \\
\hline Terminal value & 193.2 & 192.8 \\
\hline
\end{tabular}

in year 2012. Tables 1 and 2 show optimal portfolios and the magnitude of in-sample and out-of-sample mismatches. The numbers in Table 1 show which quantity (in thousands) of each asset should be bought or sold at the end of each particular year and the total initial position in year 2012. For the mismatches in Table 2, we computed the objective value of the cash-flow matching problem for both portfolios in-sample and out-of sample and divided by the fair value of liabilities. Therefore, these numbers can be viewed as a relative error.

It needs to be noted that in the terminal value matching problem, all strategies concerning purchases and sales of the cash account lead to the same objective value. Hence, the terminal position of 178.091 could have been spread in all possible manners over the years 2012-2026 without any difference.

As one may have expected, the replicating portfolio obtained from discounted terminal value matching very badly matches cash payments in particular years since these mismatches are not penalized by the objective function of the discounted terminal value matching problem. Consequently, a replicating portfolio obtained from terminal value matching is of little use to the insurer if cash payments are supposed to match well at each point in time. As already explained, the missing remedy is to employ an approximation of the appropriate dynamic investment strategy in the numéraire asset.

We implemented the linear approximation of the optimal dynamic investment strategy as outlined at the end of Sect. 4 for the same scenarios that were used for the portfolio optimizations (see Fig. 2). Table 3 shows the optimal parameters $\left(a_{t}^{1}, a_{t}^{2}, a_{t}^{3}\right)_{t=1, \ldots, T-1}$ and the coefficients of determination $R^{2}$.

On first sight, it is striking how large the coefficients of determination $\left(R^{2}\right)$ are (on average above $80 \%$ ). However, since the optimal $\delta_{t}$ is a linear combination of discounted financial cash-flows $C_{\mathrm{CA}}^{F}\left(t, D_{t}^{F}\right) / N_{t}, C_{\mathrm{SP}}^{F}\left(t, D_{t}^{F}\right) / N_{t}$ and $C_{\mathrm{NK}}^{F}\left(t, D_{t}^{F}\right) / N_{t}$ and discounted liability cash-flow $C^{L}\left(t, D_{t}^{F}, D_{t}^{L}\right) / N_{t}$, this is not too surprising. Actually, if liability cash-flows were known, i.e., available for the regression, a perfect fit (i.e., $R^{2}=100 \%$ ) would be obtainable. In all other cases, the liability cash-flow is approximated by the asset cash-flows rather well.

Analogous to Table2, Table 4 shows the in-sample and out-of-sample objective function values for the portfolio solving $\left(\mathrm{RP}_{\mathrm{CF}}\right)$ and the portfolio solving $\left(\mathrm{RP}_{\mathrm{TV}}\right)$

(Footnote 4 continued)

were generated with standard models from the Barrie and Hibbert Economic Scenario Generator (see www.barrhibb.com/economic_scenario_generator). 


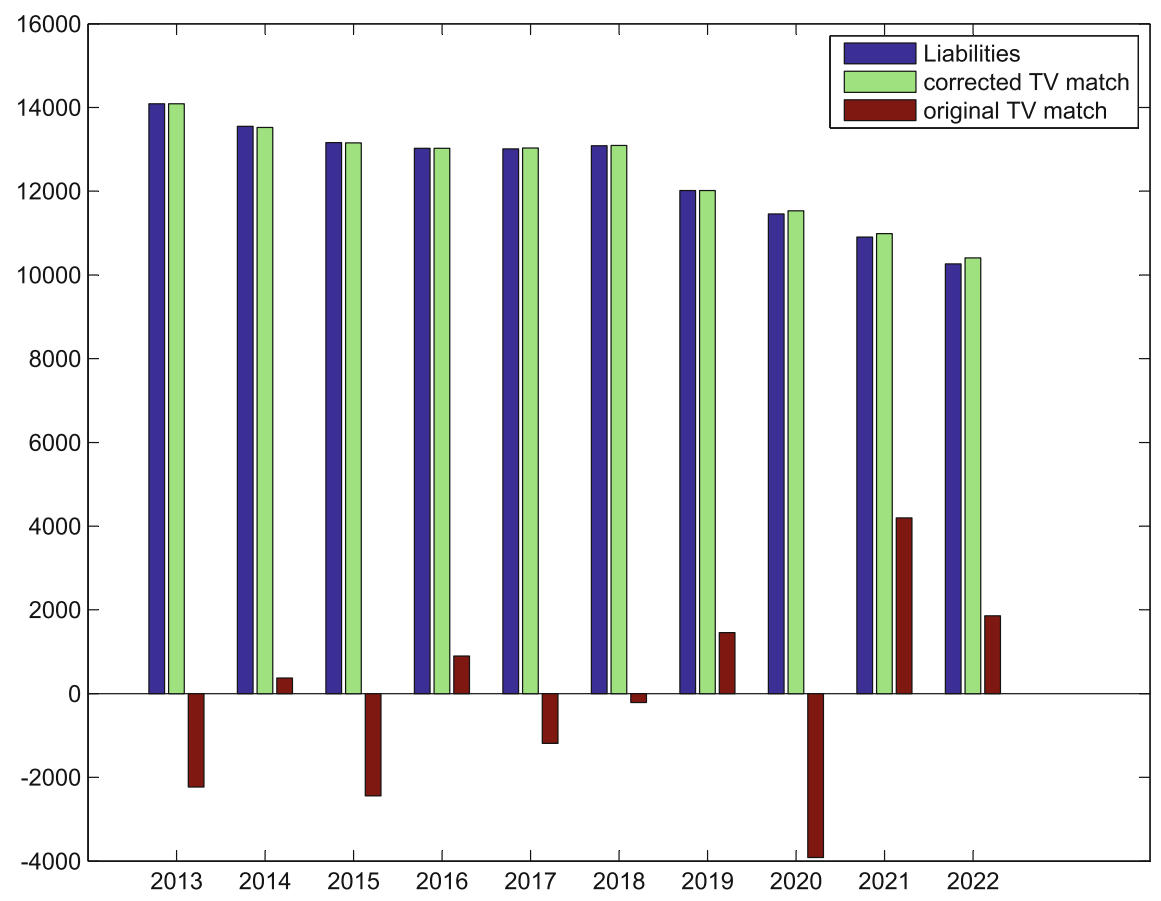

Fig. 2 The bar chart shows cash-flows of liabilities and the optimal terminal value replicating portfolio with and without a dynamic correction in the first ten years

together with dynamic investment strategy $\left(\hat{\delta}_{t}\right)_{t=1, \ldots, T}$ relative to the fair value of the liabilities.

Clearly, the dynamic strategy in the replicating assets significantly improves the quality of the cash-flow match. Yet, the optimal portfolio for cash-flow matching still slightly outperforms this dynamic variant due to the reasoning given above.

We also regressed with additional in-the-money call options on the cash-flows, but there was only a negligible improvement in-sample and out-of-sample. Possibly, one may achieve better results with a more sophisticated choice of regressors, but that seems unlikely or at least challenging given the high coefficients of determination. Further, all results obtained above have been tested to be quite stable when changing the number of scenarios or changing the specific choice of liabilities. Of course, a more detailed analysis based on a real-world example could provide further valuable insights. 
Table 3 Parameters (in thousands) obtained from linear regression and the coefficients of determination

\begin{tabular}{l|l|l|l|l}
\hline Year & $a^{1}$ & \multicolumn{1}{l}{$a^{2}$} & \multicolumn{1}{l}{$a^{3}$} & $R^{2}$ \\
\hline 2012 & 1.4089 & -2.3976 & 0.6460 & 1 \\
\hline 2013 & 1.3634 & -1.9468 & 0.3073 & 0.96 \\
\hline 2014 & 1.3361 & -3.2633 & 0.9379 & 0.99 \\
\hline 2015 & 1.2956 & 1.6990 & -0.4615 & 0.87 \\
\hline 2016 & 1.2954 & 0.8510 & 0.3424 & 0.91 \\
\hline 2017 & 1.2903 & 0.3433 & 0.0117 & 0.18 \\
\hline 2018 & 1.1768 & 1.4854 & -0.4825 & 0.83 \\
\hline 2019 & 1.1267 & -0.6920 & 0.9790 & 0.96 \\
\hline 2020 & 1.0790 & 0.1306 & -0.8758 & 0.94 \\
\hline 2021 & 1.0380 & -0.5618 & -0.2773 & 0.69 \\
\hline 2022 & 1.0143 & -1.4692 & 0.1697 & 0.61 \\
\hline 2023 & 1.0078 & -1.4899 & 0.2622 & 0.78 \\
\hline 2024 & 0.9846 & 1.3168 & -0.4793 & 0.93 \\
\hline 2025 & 0.9706 & -0.5706 & 0.0376 & 0.43
\end{tabular}

Table 4 Values of the objective function in $\left(\mathrm{RP}_{\mathrm{CF}}\right)$ for the optimal portfolio to $\left(\mathrm{RP}_{\mathrm{CF}}\right)$ and the optimal portfolio $\left(\mathrm{RP}_{\mathrm{TV}}\right)$ with strategy $\left(\hat{\delta}_{t}\right)_{t=1, \ldots, T}$ relative to the fair value of liabilities

\begin{tabular}{l|l|l}
\hline & Cash-flow $(\%)$ & T.V. w. correction $(\%)$ \\
\hline In-sample & 8.72 & 10.16 \\
\hline Out-of-sample & 9.23 & 11.05 \\
\hline
\end{tabular}

\section{Conclusion}

Motivated by the theoretical results in [7], we improved the cash-flow matching quality of the optimal terminal value portfolio without deterioration of the terminal value match. This is achieved by the introduction of a deterministic strategy (e.g., in replicating assets or risk factors) which approximates the optimal non-deterministic strategy. It turned out that with the dynamic correction the terminal value matching technique is comparable (but still slightly inferior) to the static cash-flow matching technique in terms of in-sample as well as out-of-sample performance. Due to the high coefficients of determination, a significant improvement by a more selected choice of explaining variables seems unlikely. Taking into account that in contrast to cash-flow matching, terminal value matching has an explicit analytic solution and that the least squares problems involved in the approximation of the dynamic strategy are also numerically negligible, this might thus represent a computationally more efficient alternative to the standard cash-flow matching approach. Further evidence can only be obtained by the careful examination of a real-world scenario. 
Acknowledgments The authors would like to thank Pierre Joos, Christoph Winter, and Axel Seemann for very helpful discussions and feedback. We are also grateful for the generous constant financial support by Allianz Deutschland AG. Finally, many thanks go to two anonymous referees of this paper for valuable comments which helped to improve presentation.

Open Access This chapter is distributed under the terms of the Creative Commons Attribution Noncommercial License, which permits any noncommercial use, distribution, and reproduction in any medium, provided the original author(s) and source are credited.

\section{References}

1. Andreatta, G., Corradin, S.: Fair value of life liabilities with embedded options: an application to a portfolio of Italian insurance policies. Working Paper, RAS Spa, Pianificazione Redditività di Gruppo

2. Baione, F., De Angelis, P., Fortunati, A.: On a fair value model for participating life insurance policies. Invest. Manag. Financ. Innov. 3(2), 105-115 (2006)

3. Bauer, D., Bergmann, D., Kiesel, R.: On the risk-neutral valuation of life insurance contracts with numerical methods in view. Astin Bull. 40, 65-95 (2010)

4. Dubrana, L.: A formalized hybrid portfolio replication technique applied to participating life insurance portfolios. Available at http://www.ludovicdubrana.com// (2013) Accessed 30 Dec 2013

5. Grosen, A., Jørgensen, P.L.: Fair valuation of life insurance liabilities: the impact of interest rate guarantees, surrender options, and bonus policies. Insur.: Math. Econ. 26(1), 37-57 (2000)

6. Longstaff, F., Schwartz, E.: Valuing american options by simulation: a simple least-squares approach. Rev. Financ. Stud. 14(1), 113-147 (2001)

7. Natolski, J., Werner, R.: Mathematical analysis of different approaches for replicating portfolios. Euro. Actuar. J. (2014). doi:10.1007/s13385-014-0094-Z

8. Oechslin, J., Aubry, O., Aellig, M., Kappeli, A., Bronnimann, D., Tandonnet A., Valois, G.: Replicating embedded options. Life and Pensions Risk, 47-52 (2007)

9. Pelsser, A.: Pricing and hedging guaranteed annuity options via static option replication. Insur: Math. Econ. 33(2), 283-296 (2003) 